\title{
The Low Mass End of the Young Cluster IC2391
}

\author{
David Barrado y Navascués \\ LAEFF-INTA. Apdo. 50727, 28080 Madrid. SPAIN \\ John R. Stauffer \\ IPAC, California Institute of Technology, Pasadena, CA 91125, USA
}

\begin{abstract}
By collecting optical and infrared photometry, as well as medium resolution spectroscopy, we have discovered a sample of low mass stars and brown dwarfs in the young cluster IC2391. Using the lithium depletion boundary near the substellar limit, we have estimated the age of the cluster as $\sim 50 \mathrm{Myr}$. We have also studied the $\mathrm{H} \alpha$ emission in this sample.
\end{abstract}

\section{Introduction}

During the last few years, a large amount of knowledge has been gained regarding the nature and properties of low mass stars and brown dwarfs. We have studied several nearby, young clusters, which, due to these characteristics, are excellent targets. One of these clusters is IC2391, located at $155 \mathrm{pc}-(\mathrm{m}-\mathrm{M})_{O}=5.95 \pm 0.1-$, and with a low interstellar reddening $-\mathrm{E}(B-V)=0.06$ (Patten \& Simon 1996). In previous papers, we have estimated the age of the cluster based on the lithium depletion technique ( $\tau=53 \pm 5 \mathrm{Myr}$, Barrado y Navascués et al. 1999) and identified a large number of low mass candidate members (Barrado y Navascués et al. 2001). In this paper, we present new spectroscopy of cluster members, improve the lithium age, and analyze other properties such as the $\mathrm{H} \alpha$ activity and the mass function (MF).

\section{Analysis}

\subsection{The new spectroscopic data.}

We have collected multifiber medium resolution spectroscopy with the Hydra II spectrograph at the CTIO 4m telescope in March 10th and 13th, 1999. We took several individual exposure of 1 hour each (except the last one, of half an hour), totaling 11.5 hours. Each of these exposures was processed one by one, and the individual spectrum extracted within the IRAF environment. Finally, they were combined to provide a single spectrum for each target. For each IC2391 candidate member, the 1 hour spectra and the combined spectrum were used to derive rough radial velocity, $\mathrm{H} \alpha$ and lithium equivalent widths $-\mathrm{W}(\mathrm{H} \alpha)$ and 


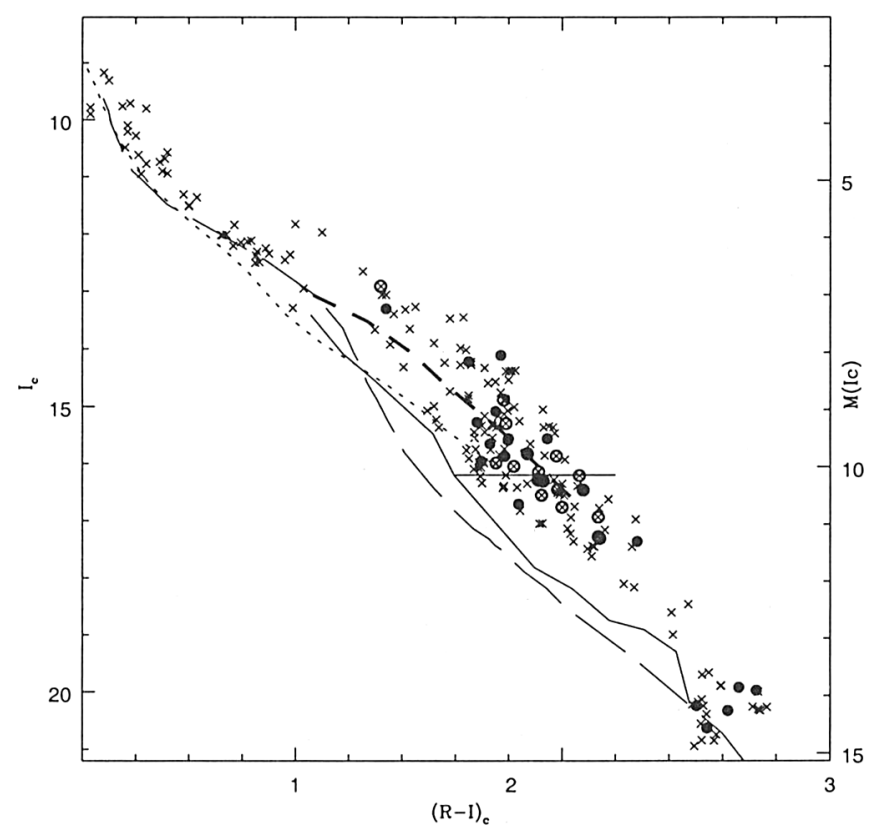

Figure 1. Color-magnitude diagram for IC2391 candidate members. Crosses represent all the available photometric data from Simon \& Patten (1998), Patten \& Pavlovsky (1999) and Barrado y Navascués et al. (2001). Open circles correspond to spectroscopic data from Barrado y Navascués et al. (1999), whereas solid circles were observed with HydraII. Several 50 Myr isochrones (see text) and an empirical zero age main sequence are also represented.

W(LiI6708 $\AA$ ). We also estimated the spectral types based on comparison with field stars and IC2391 members with previously known classification.

Figure 1 displays a color-magnitude diagram for the cluster. All known IC2391 photometric candidate members are shown as crosses, whereas circles represent objects which have spectroscopy (open and solid from Barrado y Navascués et al. 1999 and this paper, respectively).

The previously identified location of the lithium depletion boundary (LDB) is indicated with a solid horizontal segment. The new data, combined with the former spectroscopic, allow a detailed study of the depletion of lithium near the substellar limit and, hence, to improve the age estimate of the cluster based on this technique. In addition, we have collected spectra of several low mass brown dwarf candidates, with $\mathrm{M}(I c) \sim 14 \mathrm{mag}$, establishing membership in about half of them. Jameson et al. (2002) has noticed that there is a gap in the CMD in several young clusters for mid-M spectral type members. Our IC2391 data appear to support its reality.

\subsection{Lithium depletion and the age of the cluster}

Figure 2 represents the lithium equivalent widths versus the absolute $I_{C}$ magnitude and the $(R-I)_{C}$ color. For completeness, we have included data from 

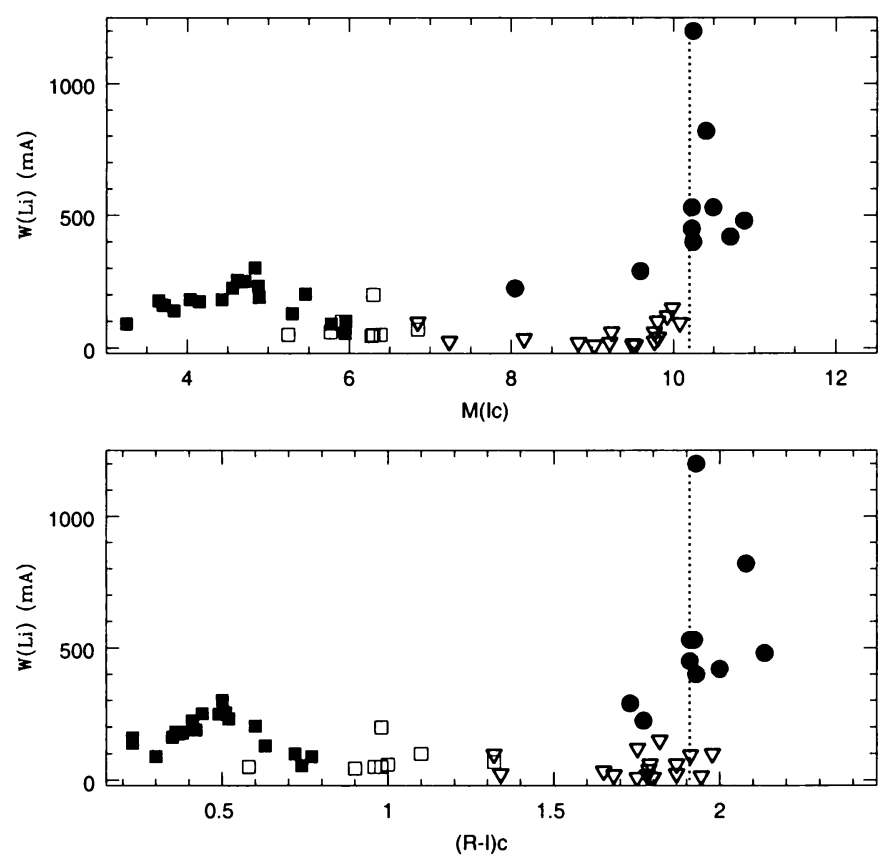

Figure 2. Lithium equivalent width versus the absolute $I c$ magnitude (a) and $(R-I) c$ color index (b). Circles and triangles represent data from this work and Barrado y Navascués et al. 1999, whereas squares correspond to data from the literature. Actual data and upper limits are displayed as solid and open symbols, respectively. The vertical dotted line locate the lithium depletion boundary for the cluster.

the literature as squares (open symbols denote upper limits. Our IC2391 data (this paper and Barrado y Navascués et al. 1999) are displayed as solid circles and open triangles (upper limits). These two panels clearly show the presence of the so-called "lithium chasm", where lithium has been completely depleted, and the location of a sharp increase in the $\mathrm{W}(\mathrm{Li})$ at the right-hand side, corresponding to the LDB (dotted line). Note that we have detected lithium in two low mass stars located to the left of the LDB. A similar situation has been found in the Pleiades cluster (Oppenheimer et al. 1997). For our two stars in IC2391, the fainter of the two is most easily explained as a nearly equal mass binary; the brighter star with $\mathrm{M}\left(\mathrm{I}_{c}\right) \sim 8.0$ cannot be explained in that fashion (and thus deserves further study). The magnitude of the LDB can be estimated as $\mathrm{M}\left(I_{C}\right)=10.15 \mathrm{mag}$.

Another color-magnitude diagram is presented in Figure 3. In this case, we have plotted the 2MASS $K s$ magnitudes against the $\left(I_{C}-K s\right)$ color index. Crosses correspond to IC2391 candidate members for which we have not obtained spectra. Open circles represent probable members with no lithium in their spectrum, whereas solid circles are probable members where we have been able to identify it. We have included a $50 \mathrm{Myr}$ isochrone by Baraffe et al. (1998) and a ZAMS (long-dashed and solid lines, respectively). As happens in Figure 2 , the LDB is clearly seen, in this case located at $\mathrm{M}(K s)=7.596$ mag. 


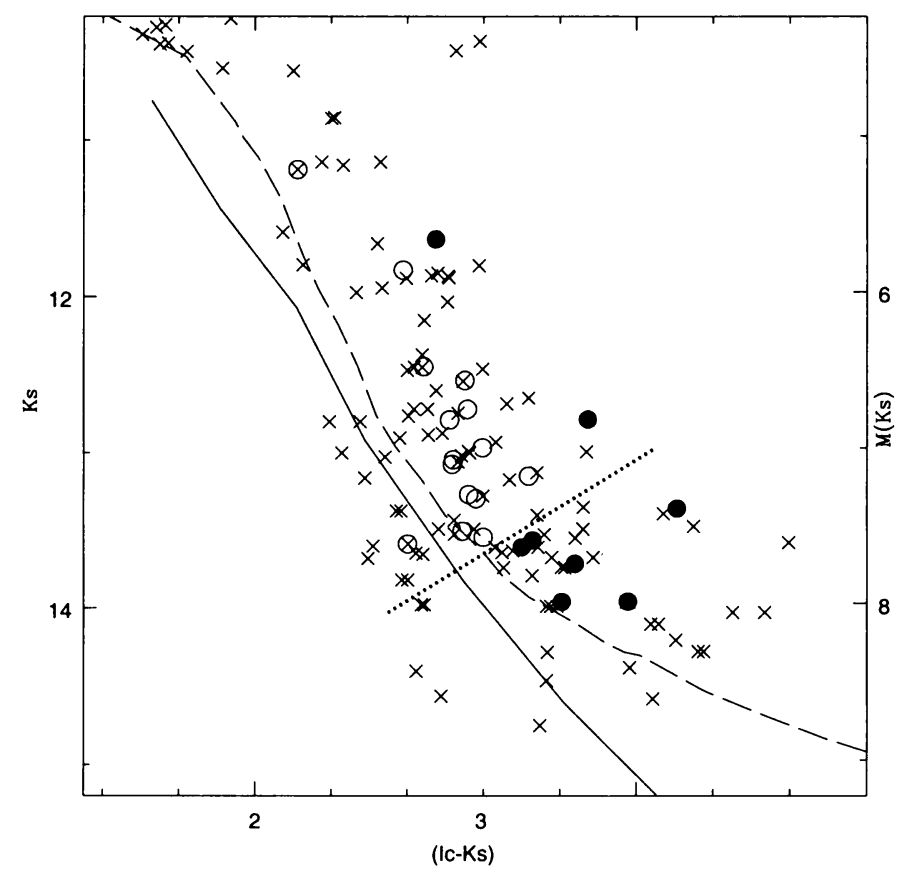

Figure 3. Color-magnitude diagram for IC2391 candidate members. Crosses represent all candidate members from Simon \& Patten (1998), Patten \& Pavlovsky (1999) and Barrado y Navascués et al. (2001). IC2391 members with lithium detection are shows as solid circles, whereas those lacking the Li6708 $\AA$ feature are displayed as open symbols. A $50 \mathrm{Myr}$ isochrones is also included (Baraffe et al. 1998, longdashed line), as well as an empirical ZAMS (solid line) and the location of the lithium depletion boundary (dotted line).

We have estimated lithium abundances for the available data. Figure 4 shows these abundances against the effective temperature. We have used the Soderblom et al. (1993) curves of growth for the stars located at the left-hand side of the "lithium chasm" and those published by Zapatero Osorio et al. (2002) for the cool end of the cluster sequence. Effective temperatures were derived based on Bessell (1979) for stars warmer than M0 and Leggett (1992) for cooler members.

Several lithium depletion isochrones by D'Antona \& Mazzitelli (1994) and Chabrier et al. (2000) are also included. Upper limits are shown as solid triangles, whereas solid circles represent actual abundances. Although these values are not very accurate for IC2391 members close to the substellar regime (due to problems such as opacities with the atmospheric models), the LDB is, again, easily visible, at about $\mathrm{T}_{\text {eff }}=3000 \mathrm{~K}$. The isochrone which better reproduce the lithium depletion pattern in the IC2391 cluster is a 50 Myr model.

So far, we have identified the location of the LDB in three open clusters, namely IC2391 (Barrado y Navascués et al. 1999 and this paper), $\alpha$ Per (Stauffer et al. 1999) and the Pleiades (Stauffer et al. 1998). The standard ages of 


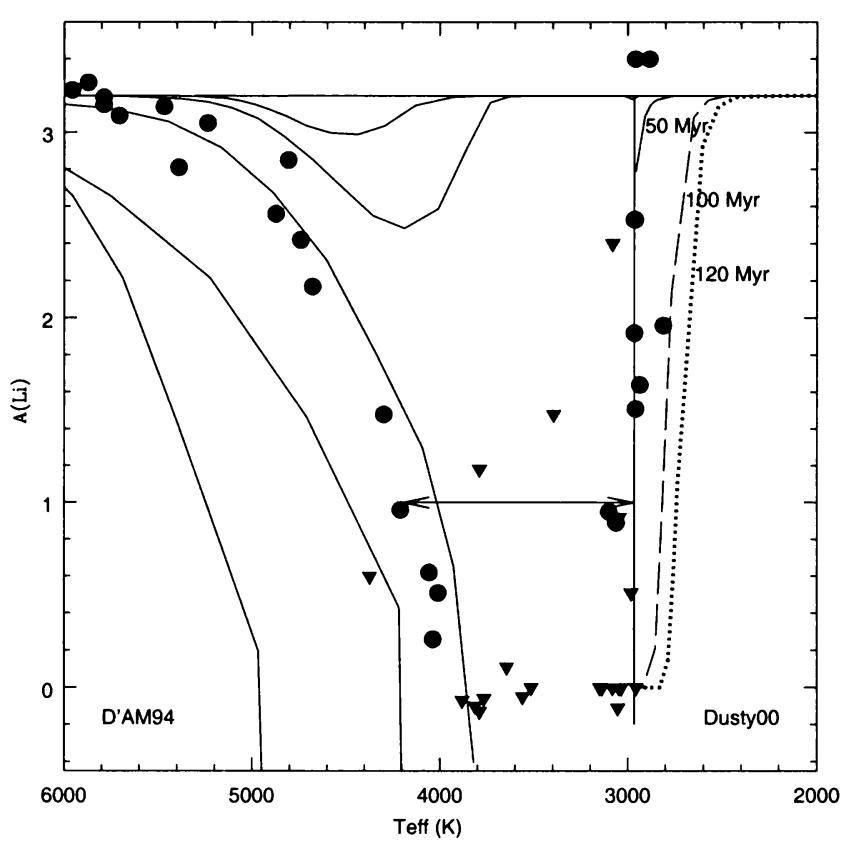

Figure 4. Lithium abundance versus effective temperature. Actual abundances and upper limits are shown as circles and triangles, respectively. Several lithium depletion isochrones from D'Antona \& Mazzitelli (1994) $-1,3,5,10,20$ and $100 \mathrm{Myr}$, left- and Chabrier et al. (2000) $-50,100$ and 120 Myr; right- are included.

these clusters, based on upper main sequence isochrone fitting are $\sim 30, \sim 50$ and 80-100 Myr, respectively. We have re-analyzed the optical and infrared available data and establish the LBD in the $\mathrm{M}\left(I_{C}\right), \mathrm{M}(K)$ and $\mathrm{M}($ bol) magnitudes. Figure 5 displays the comparison for these three magnitudes and the three clusters. The solid thick line correspond to Baraffe et al. (1998) theoretical tracks, although similar results can be achieved using Burrows et al. (1997) or D'Antona \& Mazzitelli (1994) calculations. The diagram easily shows that, indeed, the lithium ages are about $50 \%$ older than the ages derived using the location in CMD of massive stars evolving off the main sequence. The age difference may be attributable to a moderate core-overshooting in the massive stars (Ventura et al. 1998), which would increase their life inside the main sequence by a $50 \%$, though we cannot be certain yet if this is the correct explanation.

\subsection{The $\mathbf{H} \alpha$ emission}

We have investigated the $\mathrm{H} \alpha$ emission at the end of the cluster sequence and compare it with other young clusters. Figure 6 illustrates the $\mathrm{W}(\mathrm{H} \alpha)$ against the $(R-I)_{C}$ color index. IC2391 data are shown as solid circles, whereas $\sigma$ Orionis and $\alpha$ Persei data are displayed as solid triangles and crosses, respectively (see Barrado y Navascués et al. 2002 for additional information). Note that several $\sigma$ Orionis low mass stars and BDs have very large $\mathrm{W}(\mathrm{H} \alpha)$, located outside the diagram, and are not shown for clarity. Actually, some of them also have infrared 


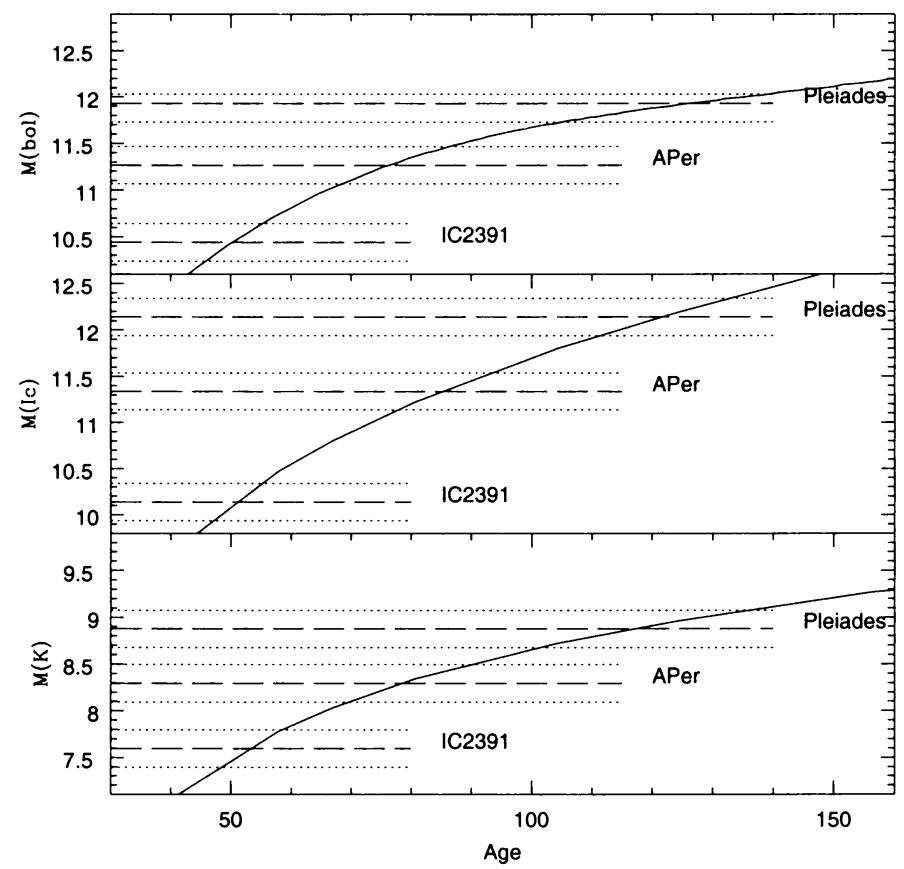

Figure 5. Location of the LBD for IC2391, Alpha Per and the Pleiades.

excesses and/or forbidden lines and have been classified as classical $\mathrm{T}$ Tauri substellar analogs (Barrado y Navascués et al. 2002). The comparison between these three clusters indicates that they have similar pattern in the $\mathrm{H} \alpha$ emission. In principal, for most of this objects, the origin of this phenomenology should be chromospheric, except in the few cases already pointed out in $\sigma$ Orionis, which is much younger than IC2391. In this last case, as in $\alpha$ Per, all the possible circumstellar disks have had enough time to dissipate.

Figure 6 shows two main features: the steadily increase of the $\mathrm{W}(\mathrm{H} \alpha)$ toward cooler objects, up to $(R-I)_{C} \sim 1.5$ (mid M spectral type), and a large dispersion for cooler objects, well within the substellar domain. Note, however, that the signal-to-noise ratio is worse for those objects, due to their faintness, and therefore, the errors in the $\mathrm{W}(\mathrm{H} \alpha)$ are larger. The enhanced spread in $\mathrm{W}(\mathrm{H} \alpha)$ for the cooler objects may also be indicative of larger or more frequent flares for these objects.

\subsection{The Mass Function}

We have derived the mass function of the IC2391 cluster and compared it with other clusters with ages ranging from $3 \mathrm{Myr}$ up to $200 \mathrm{Myr}$ ( $\sigma$ Ori, $\alpha$ Per, the Pleiades, NGC2516 and M35). Figure 7 contains these mass functions together the spectral index of a power law fit for each of them. All these MFs were derived with Baraffe et al. (1998) isochrones. Note, however, that in all cases except for IC2391 and NGC2516, we were able to remove the pollution by interlopers using additional data (infrared photometry and optical spectroscopy). Therefore, the 


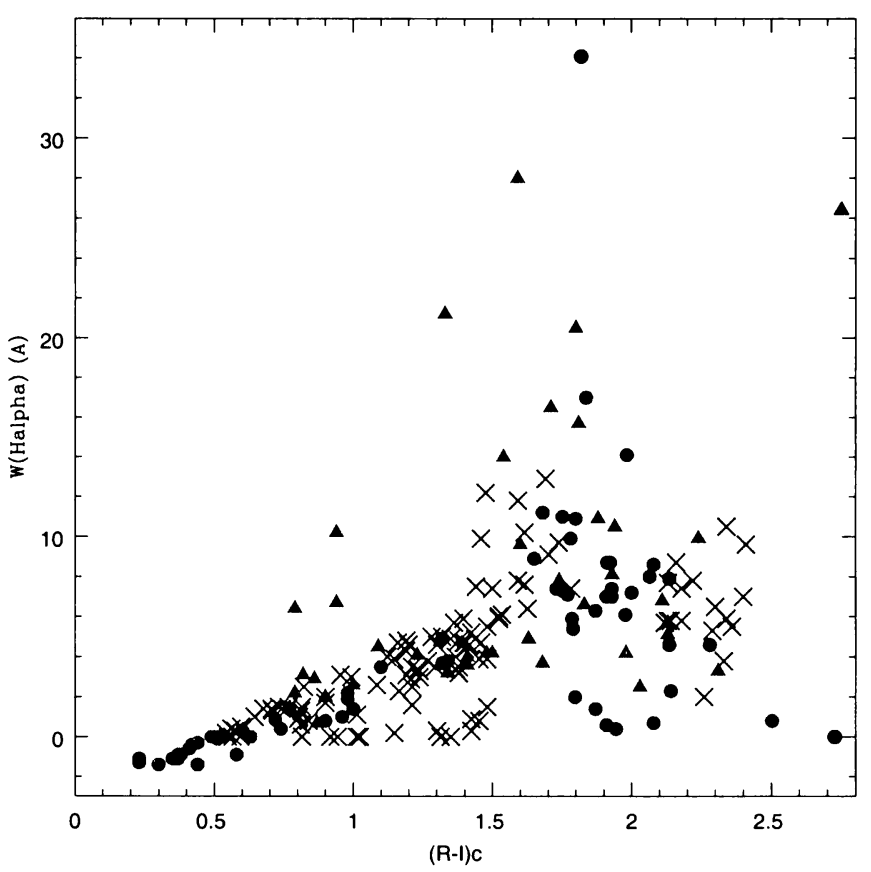

Figure 6. Comparison between the $\mathrm{H} \alpha$ equivalent widths versus the $(R-I) c$ color index for several clusters. Triangles, circles, and crosses represent data from Sigma Orionis, IC2391 and Alpha Persei clusters.

index derived for these two clusters are upper limits. In any case, although there are some differences in the structure of the MF, the general shape in these clusters is similar. The only exception is M35, a very rich cluster, where some dynamical evolution, with mass segregation, might have taken place.

\section{Conclusions.}

We have derived membership for a sample of IC2391 low mass stars and BD candidate members using medium resolution spectroscopy. In addition, by combining these data with previous spectroscopy, we have re-evaluated the LDB of the cluster and its age, which is about $50 \mathrm{Myr}$. The distribution of the $\mathrm{W}(\mathrm{H} \alpha)$ is very similar to those characteristics of younger and older clusters, as well as the cluster MF.

\section{References}

Baraffe et al. 1998, A\&A 337, 403

Barrado y Navascués et al. 1999, ApJ Letters 522, 53

Barrado y Navascués et al. 2001, ApJ Suppl. 134, 103

Barrado y Navascués et al. 2002, A\&A, in prep. 


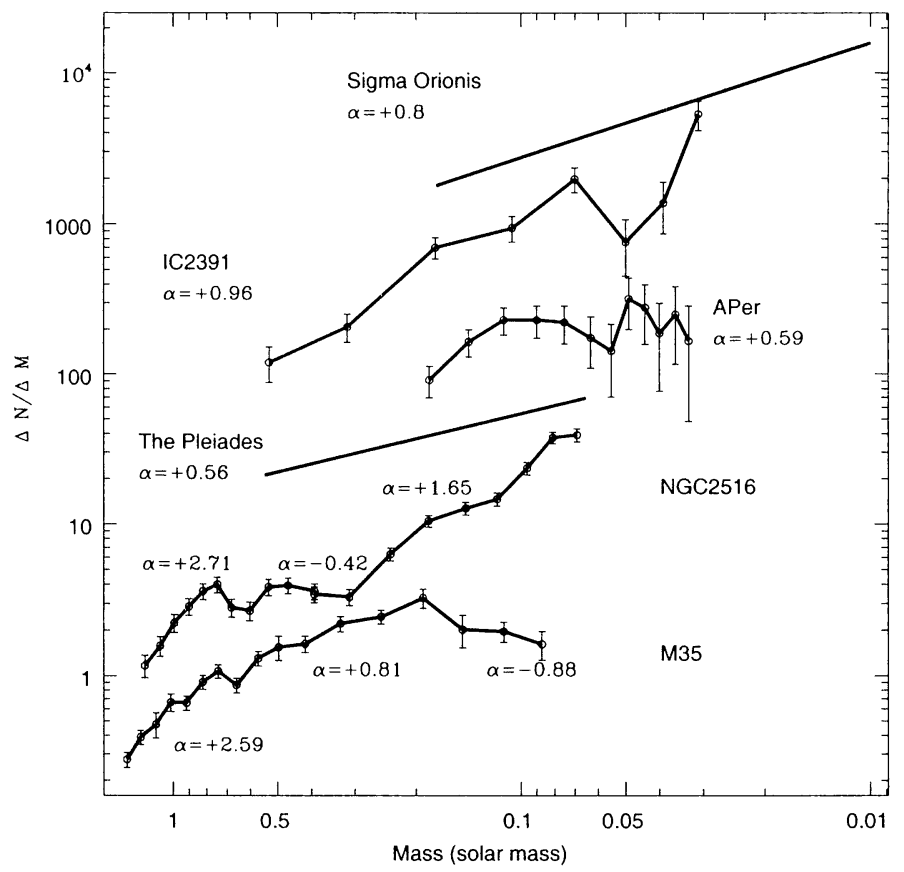

Figure 7. Mass Functions corresponding to several young open clusters.

Bessell 1979, PASP 91, 589

Burrows et al. 1997, ApJ 491, 856

Chabrier et al. 2000, ApJ 542, 464

D'Antona \& Mazzitelli, 1994, A\&A Suppl.

Gershberg et al. 1999, A\&A Suppl. 139, 555

Jameson et al. 2002, this volume

Leggett 1992, ApJ Suppl. 82, 351

Oppenheimer et al. 1997, AJ 113, 2134

Patten \& Simon, 1996, ApJ Suppl. 106, 489

Patten \& Pavlovsky, 1999, PASP 111, 210

Simon \& Patten, 1998, PASP 110, 283

Soderblom et al. 1993, AJ 106, 1059

Stauffer et al 1998, ApJ Letters

Stauffer et al. 1999, ApJ 527, 219

Ventura et al. 1998, A\&A 334, 953

Zapatero Osorio et al. 2002, A\&A 384, 937 